\title{
Original Research \\ First post-discharge heart rate and long-term prognosis in patients with acute myocardial infarction
}

\author{
Chuang $\mathrm{Li}^{1, \dagger}$, Qian Zhang ${ }^{1, \dagger}$, Dejing Feng ${ }^{1, \dagger}$, Lefeng Wang ${ }^{1}$, Xinchun Yang ${ }^{1}$, Pixiong Su${ }^{1}$, \\ Kuibao $\mathrm{Li}^{1, *}$, Mulei Chen ${ }^{1, *}$ \\ ${ }^{1}$ Heart Center and Beijing Key Laboratory of Hypertension, Beijing Chao-Yang Hospital, Capital Medical University, 10020 Beijing, China \\ *Correspondence: chenmulei5971@126.com (Mulei Chen); kuibaoli@126.com (Kuibao Li) \\ ${ }^{\dagger}$ These authors contributed equally. \\ Academic Editors: Gianluca Campo and Anindita Das \\ Submitted: 27 September 2021 Revised: 24 December 2021 Accepted: 29 December 2021 Published: 17 January 2022
}

\begin{abstract}
Background: Elevated heart rate (HR) is associated with cardiovascular mortality and other events associated with acute myocardial infarction (AMI). The heart rate after discharge is likely superior to reflect the deteriorating heart function, which negatively responds to normal physical activity. This study aimed to explore the effect of HR at the first outpatient visit on clinical outcomes. Methods: We retrospectively identified 605 patients with AMI. HRs at admission, discharge, and first outpatient visits were measured. The primary endpoint was defined as major adverse cardiovascular events (MACEs), including cardiovascular (CV) death, readmission for worsening heart failure, recurrent nonfatal myocardial infarction (MI), repeated coronary revascularization, and ischemic stroke. Results: During the follow-up period, 145 cases of MACE occurred, including $34 \mathrm{CV}$ deaths, 31 recurrent MI, 89 revascularizations, 41 heart failures, and 4 strokes. The event group displayed an elevated HR at the first outpatient visit compared to the event-free group $(p<0.001)$. After adjustment for confounding risk factors, Cox models showed that the outpatient HR had the best correlation with MACE [Hazard ratio $(\mathrm{HR})=1.33,95 \%$ confidence interval $(\mathrm{CI})=10.8-59.3, p<0.01$ for increments of 1 standard deviation (SD) in the outpatient HR) and $\mathrm{CV}$ mortality $(\mathrm{HR}=1.18,95 \% \mathrm{CI}=1.052-1.325, p<0.01)$ compared with the other two HRs. The restricted spline model indicated that $\mathrm{HR}$ at the first post-discharge above $71 \mathrm{bpm}$ was associated with CV mortality. Conclusions: Elevated HR at the first outpatient visit over a period of 2-4 weeks is related to the adverse outcomes of AMI and may identify AMI patients at higher risk of CV mortality.
\end{abstract}

Keywords: Acute myocardial infarction; Post-discharge heart rate; Mortality; MACE

\section{Introduction}

Generally, heart rate (HR) variability and fluctuation after acute and serious disorders directly reflect the severity and complexity of multiorgan dysfunction, especially in the cardiac system. Numerous previous studies have revealed that increased HR is an independent predictor of mortality in a series of cardiovascular (CV) disorders, such as acute myocardial infarction (AMI), stable coronary disease, chronic heart failure, and ischemic stroke [1-5]. Additionally, a series of evidence has been accumulated to identify that different patterns of HR, such as admission HR [6], discharge HR [3,7], HR variability [8] and resting HR [9] have been associated with a higher risk of recurrent myocardial infarction (MI) and long-term mortality in patients with AMI.

Previously, the association between elevated HR on admission and CV mortality during AMI has been recognized and incorporated into different risk stratification models, such as GRACE (Global Registry of Acute Coronary Events) and TIMI (Thrombolysis in Myocardial Infarction) risk scores [7,10]. More recent studies of patients with AMI have reported that the association between discharge HR and long-term mortality is independent and stronger than that with admission HR [1,3]. Although the effects of admission and discharge HR on long-term outcomes after MI have been well established in recent studies, few studies have investigated the effects of post-discharge HR. Meanwhile, increased HR is believed to be an indicator of more severe conditions for patients with acute coronary syndrome (ACS) [11,12], but there is limited research on the association with outpatient HR during the rehabilitation period. Therefore, this study aimed to explore the relationship between CV events and HR at the first post-discharge visit and the HR difference between discharge and the first outpatient visit (D-O diff).

\section{Materials and methods}

\subsection{Study population}

We retrospectively reviewed 6592 AMI patients undergoing primary percutaneous coronary intervention (PCI) at the Beijing Chaoyang Hospital between January 2014 and December 2019, and identified 635 patients with documented first post-discharge vital signs in the outpatient office (Supplementary Fig. 1). The diagnostic criteria for type 2 AMI were in accordance with the fourth universal definition of MI [13] when there is AMI with clinical evidence of myocardial ischemia and detection of a rise in car- 
diac troponi (cTn) values with at least above 99th percentile upper reference limit (URL) and at least one of the following: (1) typical ischemic symptoms; (2) a newly onset left bundle branch block pattern, or a new ST-segment elevation or depression in at least two contiguous leads, with findings of more than $0.2 \mathrm{mV}$ in leads $\mathrm{V} 1, \mathrm{~V} 2$, and $\mathrm{V} 3$ or at least 0.1 $\mathrm{mV}$ in the remaining leads; (3) the occurrence of pathological Q waves; and (4) new loss of viable myocardium or new regional wall motion abnormally identified by imaging evidence. The exclusion criteria were a life expectancy of less than 6 months due to cancer or cachexia, a history of coronary artery bypass inapplicable to the assessment of SYNTAX (Synergy between PCI with Taxus and Cardiac Surgery) score, liver cirrhosis, dialysis, and severe infection. Patients with atrial fibrillation and pacemakers were ruled out from this study.

\subsection{Clinical measurements and outpatient vital sign}

The admission HR and heart rhythm were simultaneously measured on an 18 lead electrocardiogram (ECG) and arm electronic sphygmomanometer (OMRON HBP-1300, Omron, Shandong, China) upon arrival to the inpatient department. The discharge HR was measured as the mean value of the last two HR values (resting in the morning before and immediately before discharge). With a routine outpatient review at 2-4 weeks after discharge, the outpatient HR was recorded in the sitting position after resting for 5min using an arm electronic sphygmomanometer (OMRON HEM-724 or HEM-1020, Omron, Shandong, China) as the mean value of the two recorded HRs with an interval of 1 -min in the department. We calculated the difference between the two above HRs, such as the D-O difference, by subtracting the first post-discharge visit HR from the value at discharge.

The patients' baseline information, including clinical features, demographics, and treatment records, were retrospectively collected from the medical database of Beijing Chaoyang Hospital. Laboratory test results, including white blood cell count, hemoglobin, platelet count, lowdensity lipoprotein, triglyceride, creatinine, fasting glucose, brain natriuretic peptide, and cardiac troponin I (CTNI), were recorded within 12-h after admission. The left ventricular ejection fraction (LVEF) performed by echocardiography within 12-h after admission was recorded. The medications were prescribed at discharge and recorded during the first post-discharge review.

In this study, two specialists (CL and DJF) were blinded to the management of medication and the outcomes during follow-up and assessed coronary angiography according to the set standards. Coronary single-vessel disease was defined as vessel stenosis $>50 \%$ in a major coronary artery or in its main branches (diameter $>1.5 \mathrm{~mm}$ ). Multiple vessel disease was defined as stenosis $>50 \%$ in two or more major coronary arteries. Subsequently, the SYNTAX score was calculated from the initial diagnostic angiogram using an online SS calculator.

This study was approved by the institutional review board of Beijing Chaoyang Hospital and performed in accordance with the 1964 Declaration of Helsinki and its later amendments. Written informed consent was obtained from all the patients or their legal relatives.

\subsection{Follow-up review and clinical outcomes}

All patients were followed up by routine outpatient visits to assess the development of the disease and the prevalence of major clinical adverse $\mathrm{CV}$ events. For patients without outpatient records, we contacted those patients by telephone to assess the incidence of $\mathrm{CV}$ events. The major adverse cardiovascular event (MACE), which is the primary endpoint of this study, was mainly defined as a composite of $\mathrm{CV}$ death, readmission for worsening heart failure, recurrent nonfatal MI, repeated coronary revascularization, and ischemic stroke.

\subsection{Statistical analysis}

Continuous variables are expressed as mean \pm standard deviation or median with interquartile range, while categorical variables are expressed as frequencies (percentages). Differences between groups were assessed by Student's $t$-test and one-way analysis of variance (ANOVA) tests for parametric data or Mann-Whitney U or KruskalWallis nonparametric tests for skewed variables. The correlation of different measures of HR with cardiac function and severity of coronary disease was also investigated. KaplanMeier survival curves were used to evaluate the incidence of MACEs, while the log-rank test was performed to determine intergroup differences among the groups. Cox proportional hazard regression analysis was performed to identify the predictors of cardiovascular mortality and MACEs. After adjusting for confounding variables, including sex, age, body mass index (BMI), and other clinical risk parameters, a restricted cubic spline curve was refabricated to present the discharge HR for CV outcomes. To explore the association of CV outcomes with different HRs, HR (95\% CI) was analyzed for standardized increments of $1 \mathrm{SD}$ of the continuous variables and presented to allow comparisons between the independent predictors. To measure the relative prognostic importance of each HR variable, HRs in hospitals and outpatients were evaluated in individual models, adjusting for the same covariates as in the original predictive model. All statistical tests were two-tailed, and the statistical significance was set at $p \leq 0.05$. Statistical analyses were performed using STATA (version 15.0, StataCorp, College Station, Texas, USA) and R statistical software (version 3.4.0, Ross Ihaka, New Zealand). 


\section{Results}

\subsection{Baseline characteristics between groups with and without MACE}

Based on the exclusion criteria, 605 AMI patients with first post-discharge vital signs were recruited for this study. The median of follow-up time was 26 months (range: 165 months). All subjects were categorized into two groups based on the occurrence of MACE. Table 1 summarizes the baseline characteristics of the two groups. The proportion of patients with and without MACE was 145 (24\%) and 460 (76\%), respectively. Interestingly, patients in the MACE group presented higher values of admission HR and outpatient HR (75 vs. 80, $p=0.03 ; 71.4$ vs. 76.2, $p<0.001$, respectively), but similar discharge $\mathrm{HR}$ (70.7 vs. 70.6, $p$ $=0.85$ ). Compared with the non-MACE group, patients with MACE were older and had a higher frequency of prior MI, history of PCI, hypertension, and hyperlipoidemia, higher levels of admission systolic blood pressure and discharge systolic blood pressure, increased incidence of Killip III or IV levels, and lower LVEF. Based on angiography features, no significant differences in the culprit vessel, number of stenosis arteries, and intra-aortic balloon pump (IABP) were observed, except for a higher SYNTAX score in the MACE group $(p=0.04)$.

\subsection{Kaplan-Meier survival curves and Cox proportional hazards regression}

During the average follow-up period of 26 months, 145 (24\%) cases with MACE occurred, including $34 \mathrm{CV}$ deaths, 31 recurrent MI, 89 revascularizations, 41 heart failures, and 4 strokes. The patients in the highest quartiles of outpatient HR presented significantly not only a higher cumulative prevalence of MACE and CV mortality, but also an increased incidence of worsening heart failure and ischemic stroke, as indicated by the Kaplan-Meier survival curves (Table 2, Fig. 1). Accordingly, the log-rank test showed significant discrepancies among the four quartiles in terms of CV mortality and MACE.

Based on the Cox proportional hazard regression analysis, the predictors of MACE and CV mortality are shown in Table 3. In the univariate analysis, it revealed that outpatient $\mathrm{HR}(\mathrm{HR}=1.03,95 \% \mathrm{CI}=1.022-1.043, p<0.001)$ and admission $\mathrm{HR}(\mathrm{HR}=1.02,95 \% \mathrm{CI}=1.002-1.040, p=$ $0.04)$, but not discharge HR $(\mathrm{HR}=0.98,95 \% \mathrm{CI}=0.942-$ $1.025, p=0.42$ ) were associated with an increased risk of mortality as well as MACE $(\mathrm{HR}=1.02,95 \% \mathrm{CI}=1.013-$ $1.031, p<0.001 ; \mathrm{HR}=1.01,95 \% \mathrm{CI}=1.002-1.022, p=$ $0.01 ; \mathrm{HR}=0.99,95 \% \mathrm{CI}=0.977-1.016, p=0.74$, respectively). After adjusting for other potential confounders, including age, sex, BMI, admission systolic pressure, administration of $\beta$-blockers, levels of hemoglobin and hs-CRP, smoking status, and a history of hypertension, diabetes mellitus, hyperlipidemia, prior MI, and PCI, it indicated that outpatient $\mathrm{HR}$ remained an independent powerful predictor of the incidence of CV mortality and MACE $(\mathrm{HR}=1.05$,
$95 \% \mathrm{CI}=1.020-1.082, p=0.001$ and $\mathrm{HR}=1.03,95 \% \mathrm{CI}=$ $1.010-1.040, p<0.01)$. Accordingly, the restricted cubic spline curve showed that the associations between HR at the first outpatient visit and MACE were approximately linear, while those with CV mortality were nonlinear (Fig. 2).

\subsection{Comparison of different measures of HR regarding $M A C E$}

As shown in Fig. 3, the standardized HRs for the six types of HRs were calculated for comparison in association with CV mortality and MACE. Each type of HR was used in a multivariate Cox regression model, including age, admission blood pressure, LVEF, prior MI, history of PCI, hypertension, diabetes mellitus, and hyperlipidemia. Notably, HR at the first outpatient visit had the strongest positive association with the occurrence of $\mathrm{CV}$ mortality and MACE $(\mathrm{HR}=1.33,95 \% \mathrm{CI}=10.8-59.3, p<0.01 ; \mathrm{HR}=1.18$, $95 \% \mathrm{CI}=1.052-1.325, p<0.01$ for increment of $1 \mathrm{SD}$ in outpatient $\mathrm{HR}$ ). In contrast, $31 \%$ risk of $\mathrm{CV}$ mortality was reduced by decreased $\mathrm{D}-\mathrm{O}$ difference $\mathrm{HR}(\mathrm{HR}=0.69,95 \%$ $\mathrm{CI}=0.565-0.846, p<0.01)$, while the D-O difference HR was nearly a predictor equal to outpatient HR for MACE $(\mathrm{HR}=0.82,95 \% \mathrm{CI}=0.726-0.931, p<0.01$ for increment of $1 \mathrm{SD}$ in D-O HR difference). In similarly adjusted models, the association with risk of mortality and MACE for admission HR was attenuated and neutral, with HR of $1.15(95 \% \mathrm{CI}=0.837-1.581, p=0.38)$ and $1.10(95 \% \mathrm{CI}=$ $0.939-1.283, p=0.24)$. Meanwhile, no association between discharge HR and A-D difference HR was found for CV mortality and MACE. Interestingly, the A-O difference HR was associated with the risk of $\mathrm{CV}$ mortality $(\mathrm{HR}=0.79$, $95 \% \mathrm{CI}=0.636-0.979, p=0.03)$, whereas no similar result was found for MACE $(\mathrm{HR}=0.91,95 \% \mathrm{CI}=0.792-1.049$, $p=0.20)$.

\section{Discussion}

Our findings suggest that the first post-discharge HR is indicative of the risk of MACE in patients with AMI. In addition, this association with CV mortality and morbidity in AMI was independent of and stronger than admission HR and discharge HR, both of which are independently related to mortality, as indicated by previous studies $[1,6]$. To the best of our knowledge, the present study is the first to illustrate the possibility that the HR at the first post-discharge visit over a recovery period of $2-4$ weeks is superior to HR at admission or discharge in predicting long-term CV outcomes in the setting of AMI.

$\mathrm{HR}$ is an essential, prognostic, and vital parameter that is very convenient to measure and monitor in clinical practice and may be applicable to therapeutic interventions in coronary artery disease. Several previous studies have established a link between the increased risk of mortality and different patterns of $\mathrm{CV}$ events and elevated resting HR [9,14-16]. Kim et al. [4] suggested that worsening the first post-discharge HR control increases the risk of read- 
Table 1. Baseline characteristic.

\begin{tabular}{|c|c|c|c|}
\hline Factor & Non-MACE group & MACE group & $p$-value \\
\hline $\mathrm{N}$ & $460(76.0 \%)$ & $145(24.0 \%)$ & \\
\hline age, (year) & $61.18(13.09)$ & $65.18(10.75)$ & $<0.001$ \\
\hline Male, n (\%) & $354(77.0 \%)$ & $109(75.2 \%)$ & 0.65 \\
\hline BMI, $\left(\mathrm{kg} / \mathrm{m}^{2}\right)$ & $25.3(3.76)$ & $25.6(3.37)$ & 0.42 \\
\hline Diagnosis, $\mathrm{n}(\%)$ & & & 0.10 \\
\hline STEMI & $448(97.4 \%)$ & $144(99.3 \%)$ & \\
\hline NSTEMI & $12(2.6 \%)$ & $1(0.7 \%)$ & \\
\hline Killip III or IV, n (\%) & $42(9.1 \%)$ & $18(12.4 \%)$ & $<0.001$ \\
\hline LVEF, \% & $62(53,67)$ & $58(48,64)$ & $<0.001$ \\
\hline Outpatient HR, (beat/min) & $70(64,75)$ & $72(68,80)$ & $<0.001$ \\
\hline Outpatient SBP, (mmHg) & $125.8(16.83)$ & $127.1(15.73)$ & 0.41 \\
\hline Outpatient DBP, $(\mathrm{mmHg})$ & $74.1(9.92)$ & $73.1(9.45)$ & 0.28 \\
\hline Admission SBP, (mmHg) & $123.1(20.0)$ & $127.8(21.14)$ & 0.02 \\
\hline Admission DBP, $(\mathrm{mmHg})$ & $71.2(12.86)$ & $72.1(12.01)$ & 0.52 \\
\hline Discharge SBP, $(\mathrm{mmHg})$ & $121.0(13.0)$ & $124.5(14.78)$ & $<0.01$ \\
\hline Discharge DBP, $(\mathrm{mmHg})$ & $70.4(9.09)$ & $71.8(8.75)$ & 0.08 \\
\hline Admission HR, (beat/min) & $75(66,84)$ & $80(69,86)$ & 0.03 \\
\hline Discharge HR, (beat/min) & $70.6(8.14)$ & $70.7(8.67)$ & 0.85 \\
\hline Discharge-out diff HR, (beat/min) & $-0(-8,6)$ & $-4(-10,4)$ & $<0.01$ \\
\hline Admi-dis diff HR, (beat/min) & $4(-4,15)$ & $6(-3,15)$ & 0.17 \\
\hline Admi-out diff HR, (beat/min) & $4(-4,14)$ & $3(-5,14)$ & 0.43 \\
\hline \multicolumn{4}{|l|}{ Medical history } \\
\hline Prior MI, n (\%) & $43(9.3 \%)$ & $27(19.0 \%)$ & $<0.01$ \\
\hline History of PCI, n (\%) & $29(6.3 \%)$ & $22(15.2 \%)$ & $<0.001$ \\
\hline Diabetes mellitus, n (\%) & $143(31.1 \%)$ & $56(38.6 \%)$ & 0.09 \\
\hline Hypertension, n (\%) & $240(52.2 \%)$ & $97(66.9 \%)$ & $<0.01$ \\
\hline Hyperlipoidemia, n (\%) & $105(22.8 \%)$ & $45(31.0 \%)$ & 0.05 \\
\hline Smoker, n (\%) & $272(59.1 \%)$ & $86(59.3 \%)$ & 0.97 \\
\hline \multicolumn{4}{|l|}{ Angiography characteristic } \\
\hline Culprit Vessel, n (\%) & & & 0.02 \\
\hline LAD, n (\%) & $178(38.7 \%)$ & $77(53.1 \%)$ & \\
\hline LCX, n (\%) & $71(15.4 \%)$ & $17(11.7 \%)$ & \\
\hline RCA, n (\%) & $209(45.4 \%)$ & $51(35.2 \%)$ & \\
\hline LM, n (\%) & $2(0.4 \%)$ & $0(0.0 \%)$ & \\
\hline Number of stenosis $>50 \%$ artery & & & 0.53 \\
\hline Single vessel, n (\%) & $11(2.4 \%)$ & $4(2.8 \%)$ & \\
\hline Two vessels, n (\%) & $90(19.6 \%)$ & $20(13.8 \%)$ & \\
\hline Three vessels, n (\%) & $349(75.9 \%)$ & $118(81.4 \%)$ & \\
\hline $\mathrm{LM}+$ three vessels, $\mathrm{n}(\%)$ & $10(2.2 \%)$ & $3(2.1 \%)$ & \\
\hline IABP, n (\%) & $188(40.9 \%)$ & $53(36.6 \%)$ & 0.35 \\
\hline SYNTAX score & $25.9(9.98)$ & $27.76(9.33)$ & 0.04 \\
\hline \multicolumn{4}{|l|}{ Laboratory test } \\
\hline White blood cell, $\left(10^{9} / \mathrm{L}\right)$ & $10.8(2.85)$ & $11.2(2.84)$ & 0.15 \\
\hline Precent of neutral cell, $(\%)$ & $79.8(10.10)$ & $79.7(9.99)$ & 0.90 \\
\hline Hemoglobin, $\left(10^{9} / \mathrm{L}\right)$ & $134.9(17.43)$ & $134.2(15.35)$ & 0.66 \\
\hline Platelet count, $\left(10^{9} / \mathrm{L}\right)$ & $216.4(58.20)$ & $215.5(54.34)$ & 0.85 \\
\hline Cholesterol, $(\mathrm{mmol} / \mathrm{L})$ & $4.6(1.06)$ & $4.6(1.12)$ & 0.95 \\
\hline High density lipoprotein, $(\mathrm{mmol} / \mathrm{L})$ & $1.09(0.29)$ & $1.13(0.29)$ & 0.10 \\
\hline
\end{tabular}


Table 1. Continued.

\begin{tabular}{lccc}
\hline Factor & Non-MACE group & MACE group & $p$-value \\
\hline Low density lipoprotein, $(\mathrm{mmol} / \mathrm{L})$ & $2.89(0.88)$ & $2.84(0.90)$ & 0.52 \\
Triglyceride, $(\mathrm{mmol} / \mathrm{L})$ & $1.60(0.92)$ & $1.61(2.70)$ & 0.91 \\
Fasting glucose, $(\mathrm{mmol} / \mathrm{L})$ & $7.9(3.74)$ & $7.78(3.30)$ & 0.74 \\
BNP, $(\mathrm{pg} / \mathrm{mL})$ & $711.9(1563.9)$ & $592.9(920.6)$ & 0.39 \\
CTNI, $(\mathrm{ng} / \mathrm{mL})$ & $63.2(85.40)$ & $75.4(114.4)$ & 0.17 \\
Hs-CRP, $(\mathrm{mg} / \mathrm{dL})$ & $4.69(2,10.9)$ & $4.24(2.47,11.17)$ & 0.47 \\
Creatinine, $(\mathrm{mmol} / \mathrm{L})$ & $81.9(40.30)$ & $78.90(21.53)$ & 0.38 \\
Medication at discharge & & & \\
Aspirin, $\mathrm{n}(\%)$ & $459(99.8 \%)$ & $145(100.0 \%)$ & 0.57 \\
Clopidogrel, $\mathrm{n}(\%)$ & $445(96.7 \%)$ & $142(97.9 \%)$ & 0.46 \\
Tirofiban, $\mathrm{n}(\%)$ & $245(53.3 \%)$ & $85(58.6 \%)$ & 0.26 \\
Ticagrelor, $\mathrm{n}(\%)$ & $14(3.0 \%)$ & $3(2.1 \%)$ & 0.54 \\
$\beta$-blocker, $\mathrm{n}(\%)$ & $288(62.7 \%)$ & $92(63.4 \%)$ & 0.88 \\
ACEI/ARB, $\mathrm{n}(\%)$ & $238(51.7 \%)$ & $72(49.7 \%)$ & 0.66 \\
Statin, $\mathrm{n}(\%)$ & $418(90.9 \%)$ & $127(87.6 \%)$ & 0.25 \\
Nitrogen, n (\%) & $150(32.6 \%)$ & $29(20.0 \%)$ & $<0.01$ \\
\hline Abbrovi)
\end{tabular}

Abbreviation: BMI, body mass index; STEMI, ST-segment elevated myocardial infraction; NSTEM, non ST-segment elevated myocardial infraction; LVEF, left ventricular ejection fraction; HR, heart rate; SBP, systolic blood pressure; DBP, diastolic blood pressure; MI, myocardial infarction; PCI, percutaneous coronary intervention; LAD, left anterior descending artery; LCX, left circumflex artery; RCA, right coronary artery; LM, left main artery coronary; IABP, intra-aortic balloon pump; BNP, brain natriuretic peptide; CTNI, cardiac troponin I; Hs-CRP, high-sensitivity C-reactive protein; ACEI, angiotensin converting enzyme inhibitors; ARB, angiotensin receptor blocker; SYNTAX, Synergy between PCI with Taxus and Cardiac Surgery.

(a)

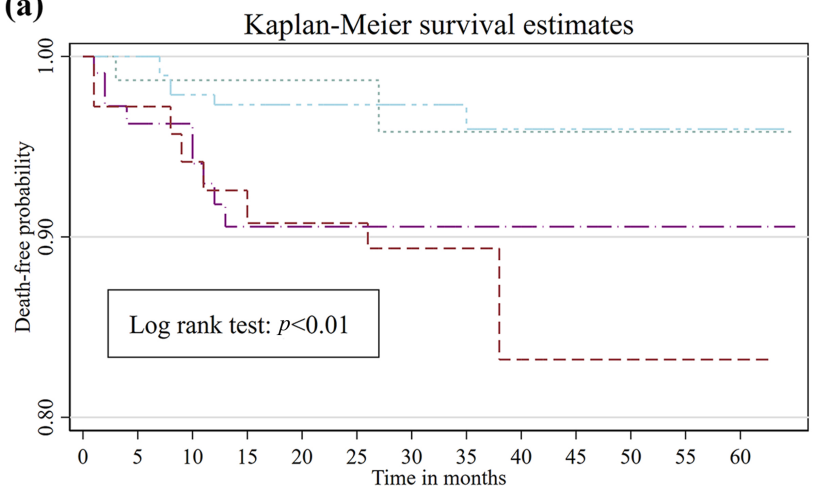

(b)

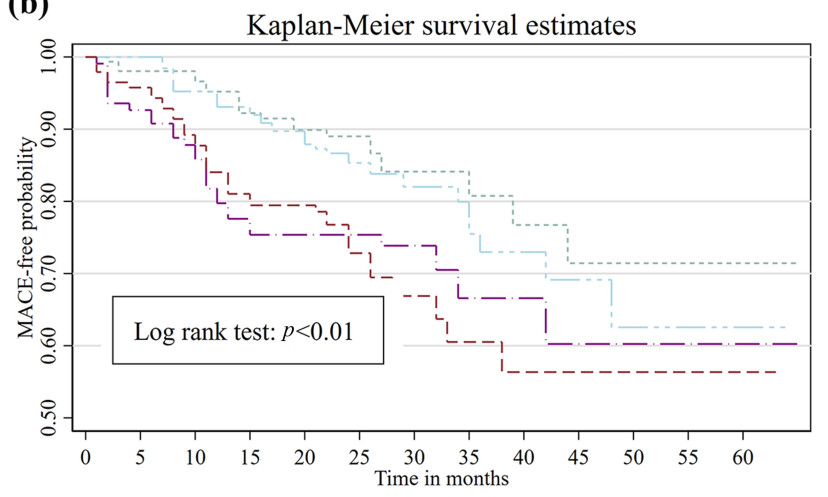

Fig. 1. The Kaplan-Meier curves for (A) CV mortality and (B) MACE among the quartiles of outpatient heart rate. The KaplanMeier curve indicated that patients in 4th quartile presented higher prevalence of CV mortality (A) and MACE (B).

mission due to the prevalence of $\mathrm{CV}$ events in patients with heart failure, including worsening heart failure, non-fatal MI, and mortality. Higher outpatient HR as a continuous parameter was related to an increased risk of mortality, with a hazard ratio of $1.037(95 \% \mathrm{CI}=1.029-1.045)$. In the case of post-MI, the early double-blind Norwegian Timolol Multicenter study, Gundersen et al. [17] demonstrated that increased resting HR at 1 month after AMI was related to mortality during a 6-years follow-up regardless of timolol treatment. Although there was no definite descrip- 
Table 2. The cumulative incidence of major adverse clinical cardiovascular events by the quartiles of outpatient heart rate.

\begin{tabular}{lccccc}
\hline & 1st quartile & 2nd quartile & 3rd quartile & 4th quartile & $p$-value \\
\hline Number, n (\%) & $153(25.3 \%)$ & $199(32.9 \%)$ & $109(18.0 \%)$ & $144(23.8 \%)$ & \\
Follow-up time, (months) & $25(19,39)$ & $27(19,39)$ & $25(11,38)$ & $24(13,33)$ & 0.02 \\
MACE, n (\%) & $25(16.3 \%)$ & $42(21.1 \%)$ & $32(29.4 \%)$ & $46(31.9 \%)$ & $<0.01$ \\
Cardiovascular mortality, n (\%) & $4(2.6 \%)$ & $6(3.0 \%)$ & $9(8.3 \%)$ & $15(10.4 \%)$ & $<0.01$ \\
Recurrent MI, n (\%) & $13(8.5 \%)$ & $7(3.5 \%)$ & $3(2.8 \%)$ & $9(6.3 \%)$ & 0.11 \\
Revascularization, n (\%) & $15(9.8 \%)$ & $33(16.6 \%)$ & $20(18.3 \%)$ & $21(14.6 \%)$ & 0.20 \\
Worsening HF, n (\%) & $4(2.6 \%)$ & $7(3.5 \%)$ & $12(11.0 \%)$ & $18(12.5 \%)$ & $<0.001$ \\
Ischemia stroke, n (\%) & $0(0.0 \%)$ & $0(0.0 \%)$ & $0(0.0 \%)$ & $4(2.8 \%)$ & $<0.01$ \\
\hline
\end{tabular}

Abbreviation: MACE, major adverse cardiovascular events; MI, myocardial infarction; HF, Heart Failure.

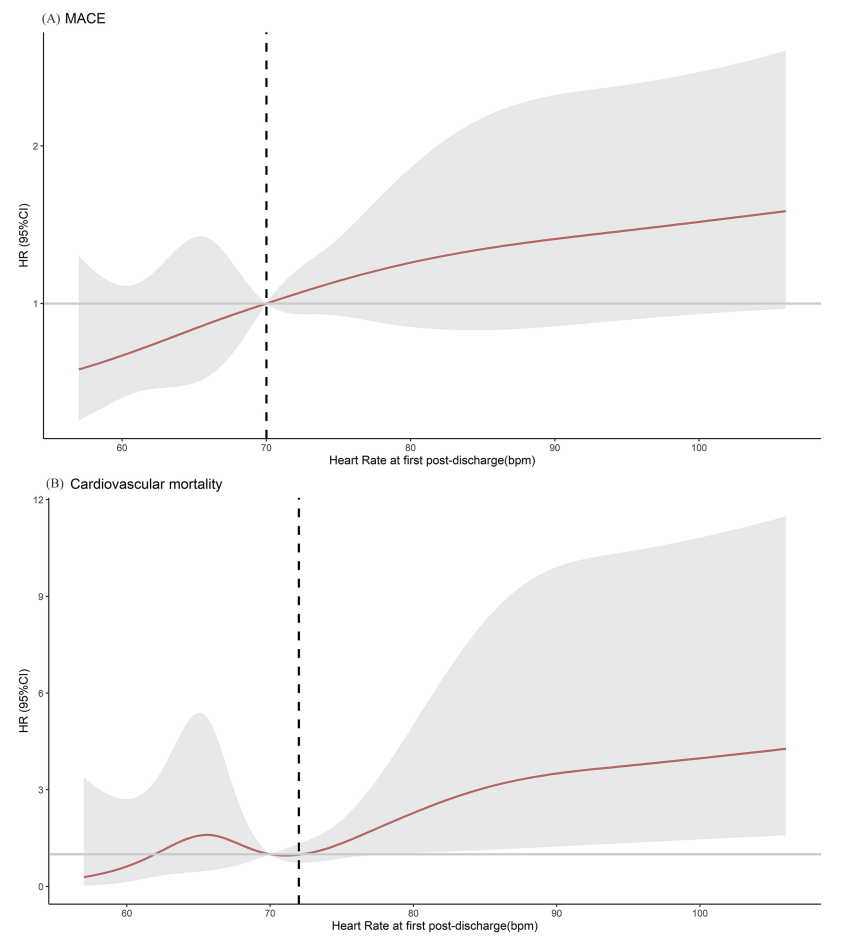

Fig. 2. Restricted cubic spline curve between outpatient HR and $\mathrm{CV}$ events and mortality presented as a rough linear relationship. (A) The linear relationship between HR at first postdischarge and $\mathrm{CV}$ events. The dashed grey area represents the upper and lower $95 \%$ confidence limits. (B) The curve (solid red) shows that a resting HR of $62-71$ beats/min was not associated with an elevated risk for $\mathrm{CV}$ mortality relative to a resting $\mathrm{HR}$ of 61 beats/min.

tion of the post-discharge HR, the failure of HR management in the second or fourth week after discharge was related to a poor prognosis. Our findings are consistent with these reports and suggest that the first post-discharge HR is a better predictor of long-term CV events in AMI patients compared with the two other types of HR at hospitalization, independent of the coronary severity and LVEF on admission. Hence, the present study provides important prognostic evidence for the prediction of early follow-up outpatient
HR in the AMI population. Additionally, increased differences between outpatient and discharge HRs were also independent risk factors for $\mathrm{CV}$ events. Although causality in this observational study cannot be assessed, existing studies provide a rational explanation. In the Coronary Artery Risk Development in Young Adults (CARDIA) Study, the increment of change in HR during follow-up visits is associated with worsening diastolic function in young adults at a higher risk of incident HF and CV disease [16]. Unrevealed diastolic dysfunction is an important independent predictor of mortality after AMI, regardless of LVEF [18]. This interaction may be a confounding factor between the elevated outpatient $H R$ and mortality. In addition, a reduction in HR potentially reduces myocardial oxygen consumption and maintains coronary perfusion due to a prolonged diastolic period and vice versa [19]. A cascade of events, including elevated left ventricular afterload and stiffness, impairments in left ventricular diastolic function, and cardiac function, are induced by an increase in HR [20].

Interestingly, contrary to the positive results from prior studies regarding the effect of discharge $\mathrm{HR}$ on $\mathrm{CV}$ mortality, our findings showed that there is a neutral association with clinical outcomes. It is worth noting that the HR at discharge was limited to approximately 70 beats/min in this population. In a recently published study [1], the findings of Alapati et al. [1] also demonstrated that discharge HR $<70 \mathrm{bpm}$ has a comparable risk of all-cause mortality compared with $\mathrm{HR}<60 \mathrm{bpm}$ with a hazard ratio of 0.95 . This is consistent with previously published data from two other large-scale studies [2,7] concerning discharge HR. Hence, in the era of timely reperfusion, the effects of $\beta$-blockers on long-term prognosis in post-MI patients with HR $<70 \mathrm{bpm}$ remains debated [21]. Accordingly, our study found that an outpatient HR $>71 \mathrm{bpm}$ was associated with CV mortality in the setting of ACS. Meanwhile, our observation suggested that resting HR in the first visit after discharge rebounded following the recovery of normal physical activity. In the case of post-discharge, activation of the sympathetic nervous system increases further due to normal physical activity, and a hemodynamically unstable phase is expected compared with hospital- 
(a) Cardiovascular mortality Outpatient HR

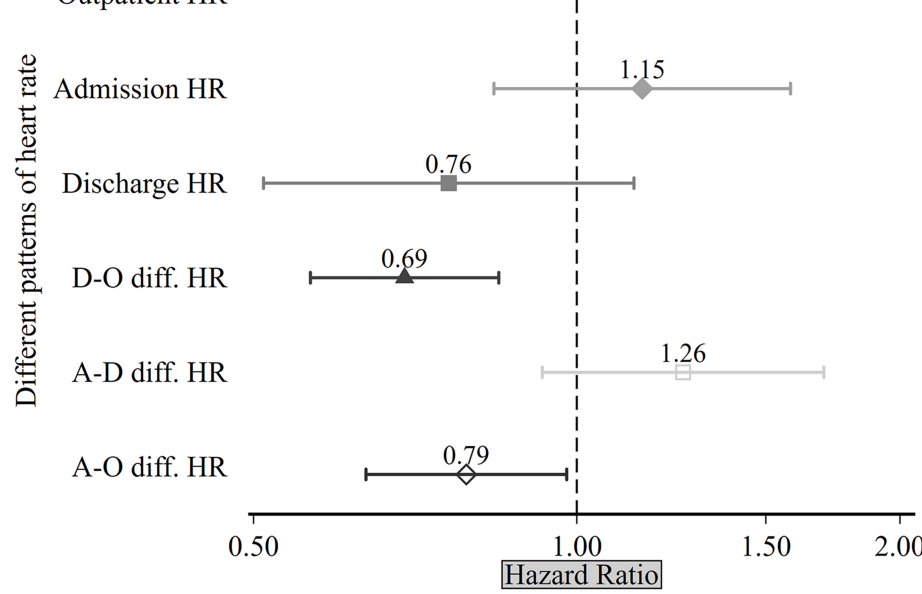

95\% Confidence Interval $\quad p_{\text {value }}$

$\begin{array}{ll}(1.108-1.593) & <0.01\end{array}$

$\begin{array}{ll}(0.837-1.581) & 0.38\end{array}$

$\begin{array}{ll}(0.511-1.131) & 0.18\end{array}$

$(0.565-0.846) \quad<0.001$

$(0.929-1.699) \quad 0.14$

$\begin{array}{ll}(0.636-0.979) & 0.03\end{array}$ (b) MACE

Outpatient HR

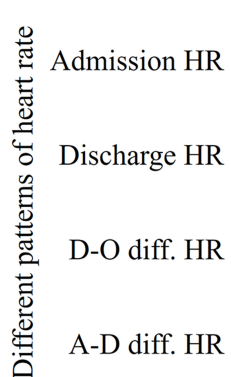

A-O diff. HR
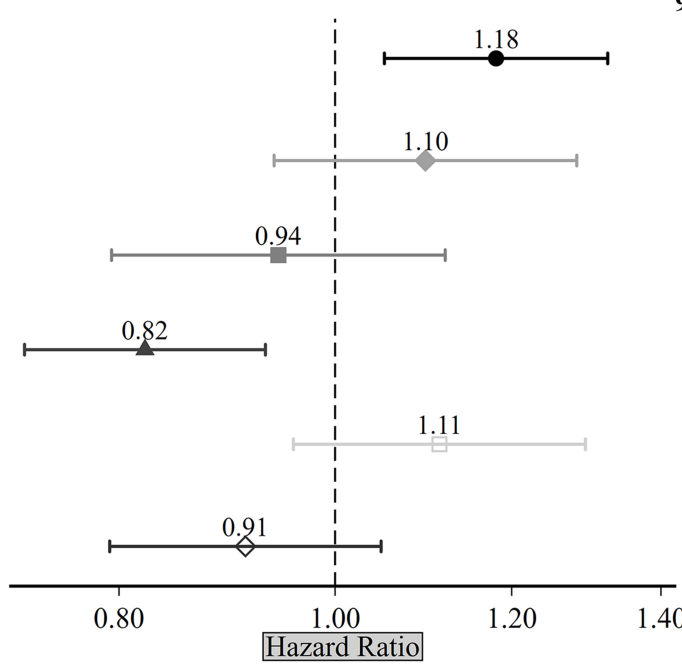

95\% Confidence Interval $p_{\text {value }}$

$\begin{array}{ll}(1.052-1.325) & <0.01\end{array}$

$\begin{array}{ll}(0.939-1.283) & 0.24\end{array}$

$\begin{array}{ll}(0.794-1.120) & 0.51\end{array}$

$(0.726-0.931) \quad<0.01$

$(0.957-1.295) \quad 0.16$

$(0.792-1.049) \quad 0.20$

Fig. 3. Comparison of effects between different patterns of heart rate or difference in heart rate on cardiovascular mortality and MACE, respectively. After adjustment for age, admission blood pressure, left ventricular ejection fraction, prior MI, and the history of PCI, hypertension, diabetes mellitus, and hyperlipemia, the outpatient heart rate showed higher risk of CV mortality and MACE, with hazard ratio (HR) of 1.33 and 1.18, respectively. Data from Cox analyses are shown and are expressed as HR (95\% CI) for Cox analysis, estimated for increments of $1 \mathrm{SD}$ in each predictive variable.

ization. The condition, referred to as post-hospital syndrome declared by Harlan, suggests that the risks in the critical 30-day period after discharge might be derived from physiological stress and mood anxiety in different scenarios, such as decompensated heart failure and acute coronary disease [22-24]. During hospitalization, a third of patients commonly experience barren sleep, disruption of circadian rhythms, moderate/severe anxiety, and reduction of activity endurance, which can adversely affect sympathetic activity and contribute to diminished physical performance [24]. Among the 7599 patients with chronic heart failure enrolled in the CHARM (candesartan in heart failure: assessment of reduction in mortality and morbidity) research [25], the up- ward changes in outpatient HR in the three months preceding visit could predict readmission and cardiac death, independent of the series of HR-reducing interventions. A recent study by Ryuichi et al. [26] also indicated that an early follow-up at outpatient management within 2 weeks could improve the long-term rehospitalization rate and composite adverse endpoints, including all-cause mortality in the setting of chronic heart failure. Early follow-up care could consecutively enforce self-management during hospitalization, resulting in an improved prognosis. In the current study, the controlled HR at discharge could contribute to the small number of irresistible events, whereas it remains possible to underestimate the condition of cardiac recov- 
Table 3. Cox proportional hazard regression analyses for cardiovascular mortality and MACE.

\begin{tabular}{|c|c|c|c|c|c|c|c|c|}
\hline & \multicolumn{4}{|c|}{ Cardiovascular mortality } & \multicolumn{4}{|c|}{ MACE } \\
\hline & \multicolumn{2}{|c|}{ Univariate analysis } & \multicolumn{2}{|c|}{ Multivariable analysis } & \multicolumn{2}{|c|}{ Univariate analysis } & \multicolumn{2}{|c|}{ Multivariable analysis } \\
\hline & $\operatorname{HR}(95 \% \mathrm{CI})$ & $p$-value & HRs $(95 \% \mathrm{CI})$ & $p$-value & $\operatorname{HR}(95 \% \mathrm{CI})$ & $p$-value & HRs $(95 \% \mathrm{CI})$ & $p$-value \\
\hline Age & $1.04(1.014-1.076)$ & $<0.01$ & $1.03(0.987-1.065)$ & 0.20 & $1.02(1.007-1.035)$ & $<0.01$ & $1.01(0.996-1.030)$ & 0.13 \\
\hline Male & $1.39(0.666-2.915)$ & 0.38 & $1.51(0.506-2.963)$ & 0.65 & $1.14(0.783-1.663)$ & 0.49 & $1.02(0.643-1.616)$ & 0.94 \\
\hline BMI & $1.10(1.024-1.188)$ & $<0.01$ & $1.1(1.033-1.269)$ & 0.01 & $1.01(0.975-1.060)$ & 0.44 & $1.03(0.977-1.076)$ & 0.30 \\
\hline Outpatient HR & $1.03(1.022-1.043)$ & $<0.001$ & $1.05(1.020-1.082)$ & 0.001 & $1.02(1.013-1.031)$ & $<0.001$ & $1.03(1.010-1.040)$ & $<0.01$ \\
\hline Admission SBP & $1.02(1.007-1.038)$ & $<0.01$ & $1.03(1.013-1.055)$ & 0.001 & $1.01(1.004-1.019)$ & $<0.01$ & $1.02(1.006-1.024)$ & $<0.01$ \\
\hline Discharge SBP & $1.01(0.991-1.036)$ & 0.26 & & & $1.01(1.000-1.021)$ & 0.04 & & \\
\hline Admission HR & $1.02(1.002-1.040)$ & 0.03 & & & $1.01(1.002-1.022)$ & 0.01 & & \\
\hline Discharge HR & $0.98(0.942-1.025)$ & 0.42 & & & $0.99(0.977-1.016)$ & 0.74 & & \\
\hline Adm-dis HR & $1.02(1.004-1.038)$ & 0.02 & & & $1.01(1.001-1.020)$ & 0.02 & & \\
\hline Adm-out HR & $0.98(0.963-0.991)$ & 0.001 & & & $0.99(0.984-1.003)$ & 0.21 & & \\
\hline Dis-out HR & $0.96(0.954-0.976)$ & $<0.001$ & & & $0.98(0.970-0.988)$ & $<0.001$ & & \\
\hline Nitrogen & $0.59(0.255-1.349)$ & 0.01 & & & $0.58(0.386-0.874)$ & 0.01 & & \\
\hline$\beta$-blocker & $0.67(0.332-1.333)$ & 0.25 & $0.91(0.392-2.092)$ & 0.82 & $1.04(0.737-1.462)$ & 0.83 & $0.99(0.686-1.435)$ & 0.97 \\
\hline Creatinine & $1.00(0.996-1.010)$ & 0.36 & $1.00(0.991-1.011)$ & 0.81 & $0.99(0.991-1.002)$ & 0.33 & $0.99(0.992-1.004)$ & 0.57 \\
\hline $\mathrm{BNP}$ & $0.99(0.999-1.000)$ & 0.84 & & & $1.00(0.999-1.102)$ & 0.24 & & \\
\hline CTNI & $1.00(0.998-1.005)$ & 0.38 & & & $1.00(1.000-1.003)$ & 0.09 & & \\
\hline $\mathrm{EF}$ & $0.95(0.917-0.976)$ & $<0.001$ & $0.95(0.910-0.990)$ & 0.02 & $0.96(0.950-0.980)$ & $<0.001$ & $0.97(0.950-0.986)$ & $<0.001$ \\
\hline Hemoglobin & $1.00(0.979-1.022)$ & 0.98 & $0.99(0.968-1.015)$ & 0.47 & $0.99(0.988-1.008)$ & 0.66 & $0.99(0.983-1.005)$ & 0.33 \\
\hline Hs-CRP & $1.00(1.002-1.004)$ & $<0.001$ & $1.00(1.003-1.006)$ & $<0.001$ & $0.99(0.976-1.021)$ & 0.91 & $0.98(0.943-1.018)$ & 0.30 \\
\hline Current smoker & $1.32(0.636-2.738)$ & 0.46 & $3.81(1.465-9.929)$ & $<0.01$ & $0.99(0.712-1.386)$ & 0.97 & $1.31(0.887-1.943)$ & 0.17 \\
\hline Hypertension & $2.67(1.209-5.901)$ & 0.02 & $1.10(0.447-2.720)$ & 0.83 & $1.68(1.192-2.381)$ & 0.02 & $1.15(0.784-1.695)$ & 0.47 \\
\hline Diabetes mellitus & $1.78(0.905-3.505)$ & 0.09 & $2.11(0.994-4.507)$ & 0.05 & $1.50(1.071-2.094)$ & 0.04 & $1.28(0.896-1.843)$ & 0.17 \\
\hline Hyperlipemia & $1.76(0.883-3.531)$ & 0.11 & $1.62(0.690-3.802)$ & 0.27 & $1.20(0.850-1.722)$ & 0.29 & $1.03(0.692-1.537)$ & 0.88 \\
\hline Prior MI & $3.92(1.912-8.052)$ & 0.001 & $2.85(1.110-7.331)$ & 0.03 & $1.99(1.306-3.019)$ & 0.001 & $1.63(1.017-2.626)$ & 0.04 \\
\hline History of PCI & $3.86(1.741-8.543)$ & $<0.001$ & $3.96(1.380-11.413)$ & 0.01 & $2.53(1.609-3.997)$ & $<0.001$ & $2.22(1.342-3.691)$ & 0.01 \\
\hline SYNTAX score & $1.02(0.989-1.049)$ & 0.22 & & & $1.013(0.998-1.027)$ & 0.08 & & \\
\hline
\end{tabular}

Abbreviation: BMI, body mass index; BNP, brain natriuretic peptide; EF, ejection fraction; PCI, percutaneous coronary intervention; HR, heart rate; SBP, systolic blood pressure; CTNI, cardiac troponin I; MI, myocardial infarction. 
ery and response to medication. Hence, particular attention needs to be paid to the importance of early follow-up outpatient care, including HR lowering, which could facilitate the management and monitoring of normal daily life habits in the early recovery period.

Our observations suggest that monitoring HR at the first post-discharge visit may be beneficial in identifying AMI patients at the greatest risk of readmission and death. The mechanism beyond the association between outpatient $\mathrm{HR}$ and death may relate to a long-term risk of $\mathrm{CV}$ autonomic neuropathy in association with various pathogenic pathways, including chronic inflammatory and atherosclerosis, and infectious disease processes. Reduction of HR variability and baroreflex gain reflect intrinsic autonomic abnormalities, which are generally associated with an imbalance of the autonomic nervous system, including sympathetic hyperactivity and vagal hypoactivity. Therefore, resting tachycardia commonly manifests after AMI, and an exaggerated HR relative to the activation of the sympathetic tone is general [27,28]. Meanwhile, a recent study by Lai et al. [29] indicated that the CV autonomic neuropathy precipitated by diabetes mellitus could contribute to sympathetic predominance and is strongly associated with subsequent major adverse $\mathrm{CV}$ events, independent of underlying coronary disease and other risk factors. Several other studies have also shown that autonomic dysfunction, defined as an alteration in vagal and sympathetic activities, is related to long-term CV events [30,31]. Moreover, higher HR is also associated with cardiometabolic factors, such as increased oxidative stress, inflammation markers, glucose intolerance, and diabetes mellitus [28,32]. In the early stage of cardiac resilience following AMI, the discrepancy regarding suboptimal lifestyle habits and CV disease burden and subsequent alterations in myocardial function are also possible contributory factors for uncontrolled resting HR in the clinic department $[16,22]$.

There were several limitations to this retrospective study. First, the research is observational, and as such, cannot establish a cause. Nevertheless, these findings are consistent with those of other studies $[4,17]$. Second, there was a low rate of $\mathrm{CV}$ events in the present study. Standard guideline-directed medical therapy can contribute to a low incidence of $\mathrm{CV}$ deaths. Third, the status of chronic inflammatory diseases, such as pulmonary disease, was not depicted in detail in this study. In the Cox proportional hazard regression, when the level of hs-CRP was considered, outpatient HR was an independent risk factor for CV mortality and MACE. Finally, because there was no difference in discharge of $\beta$-blockers between the two subgroups, we did not discuss the influence of outpatient $\beta$-blocker treatment on the effectiveness of discharge HR for long-term $\mathrm{CV}$ events. We did not report the details of patients who received $\beta$-blockers in the clinic department. Further research on this relationship should be conducted.

\section{Conclusions}

In the case of AMI, HR monitoring at the first postdischarge visit is an important parameter associated with $\mathrm{CV}$ events. During the first outpatient visit, an HR above 71 beats/min was a risk factor related to the occurrence of CV mortality and other events. Hence, a thorough followup of HR changes in outpatients with AMI may facilitate the management of patients at a higher risk of $\mathrm{CV}$ events.

\section{Author contributions}

CL, DJF and QZ conceived the present study, participated in the design, collected and assembled all data, conducted data analysis, and drafted the manuscript. PXS commented on the manuscript drafts. LFW and XCY provided material and technical support and commented on the manuscript drafts. KBL and MLC aided the interpretation of data, commented on this study design, and provided a critical review. All authors have read and approved the manuscript.

\section{Ethics approval and consent to participate}

This study was approved by the institutional review board of Beijing Chaoyang Hospital (2017-S-187) and performed in accordance with the 1964 Declaration of Helsinki and its later amendments. Written informed consent was obtained from all the patients or their legal relatives.

\section{Acknowledgment}

We would like to express our gratitude to all those who helped us during the writing of this manuscript. We thank all the peer reviewers for their opinions and suggestions.

\section{Funding}

This research was funded by the National Key R\&D Program of China, grant number 2016YFC1301102. It funded the collection of data in the outpatient department and the revision of the manuscript.

\section{Conflict of interest}

The authors declare no conflict of interest.

\section{Supplementary material}

Supplementary material associated with this article can be found, in the online version, at https://www.imrpre ss.com/journal/RCM/23/1/10.31083/j.rcm2301024.

\section{References}

[1] Alapati V, Tang F, Charlap E, Chan PS, Heidenreich PA, Jones $\mathrm{PG}$, et al. Discharge Heart Rate after Hospitalization for Myocardial Infarction and Long-Term Mortality in 2 us Registries. Journal of the American Heart Association. 2019; 8: e010855.

[2] Antoni ML, Boden H, Delgado V, Boersma E, Fox K, Schalij MJ, et al. Relationship between discharge heart rate and mortality in patients after acute myocardial infarction treated with pri- 
mary percutaneous coronary intervention. European Heart Journal. 2012; 33: 96-102.

[3] Jensen MT, Kaiser C, Sandsten KE, Alber H, Wanitschek M, Iversen $\mathrm{A}$, et al. Heart rate at discharge and long-term prognosis following percutaneous coronary intervention in stable and acute coronary syndromes - results from the BASKET PROVE trial. International Journal of Cardiology. 2013; 168: 3802-3806.

[4] Kim T, Kim H, Kim I, Yoon H, Park H, Cho Y, et al. Heart rate at first postdischarge visit and outcomes in patients with heart failure. Heart. 2018; 104: 1086-1092.

[5] Kuo Y, Lee M, Huang Y, Lee J. Initial in-hospital heart rate is associated with three-month functional outcomes after acute ischemic stroke. BMC Neurology. 2021; 21: 222.

[6] Noman A, Balasubramaniam K, Das R, Ang D, Kunadian V, Ivanauskiene $\mathrm{T}$, et al. Admission heart rate predicts mortality following primary percutaneous coronary intervention for STelevation myocardial infarction: an observational study. Cardiovascular Therapeutics. 2013; 31: 363-369.

[7] Seronde MF, Geha R, Puymirat E, Chaib A, Simon T, Berard L, et al. Discharge heart rate and mortality after acute myocardial infarction. The American Journal of Medicine. 2014; 127: 954 962.

[8] La Rovere MT, Bigger JT, Marcus FI, Mortara A, Schwartz PJ. Baroreflex sensitivity and heart-rate variability in prediction of total cardiac mortality after myocardial infarction. ATRAMI (Autonomic Tone and Reflexes after Myocardial Infarction) Investigators. Lancet. 1998; 351: 478-484.

[9] Lindgren M, Robertson J, Adiels M, Schaufelberger M, Åberg $\mathrm{M}$, Torén $\mathrm{K}$, et al. Resting heart rate in late adolescence and long term risk of cardiovascular disease in Swedish men. International Journal of Cardiology. 2018; 259: 109-115.

[10] Granger CB, Goldberg RJ, Dabbous O, Pieper KS, Eagle KA, Cannon CP, et al. Predictors of hospital mortality in the global registry of acute coronary events. Archives of Internal Medicine. 2003; 163: 2345-2353.

[11] Jensen MT, Pereira M, Araujo C, Malmivaara A, Ferrieres J, Degano IR, et al. Heart rate at admission is a predictor of inhospital mortality in patients with acute coronary syndromes: Results from 58 European hospitals: the European Hospital Benchmarking by Outcomes in acute coronary syndrome Processes study. European Heart Journal. Acute Cardiovascular Care. 2018; 7: 149-157.

[12] Zhang D, Wang W, Li F. Association between resting heart rate and coronary artery disease, stroke, sudden death and noncardiovascular diseases: a meta-analysis. Canadian Medical Association Journal. 2017; 188: E384-E392.

[13] Thygesen K, Alpert JS, Jaffe AS, Chaitman BR, Bax JJ, Morrow DA, et al. Fourth Universal Definition of Myocardial Infarction (2018). Journal of the American College of Cardiology. 2018; 72: 2231-2264.

[14] Aune D, Sen A, ó'Hartaigh B, Janszky I, Romundstad PR, Tonstad S, et al. Resting heart rate and the risk of cardiovascular disease, total cancer, and all-cause mortality - a systematic review and dose-response meta-analysis of prospective studies. Nutrition, Metabolism, and Cardiovascular Diseases. 2017; 27: 504517.

[15] Cucherat M. Quantitative relationship between resting heart rate reduction and magnitude of clinical benefits in post-myocardial infarction: a meta-regression of randomized clinical trials. European Heart Journal. 2007; 28: 3012-3019.

[16] Nwabuo CC, Appiah D, Moreira HT, Vasconcellos HD, Aghaji QN, Ambale-Venkatesh B, et al. Temporal Changes in Resting Heart Rate, Left Ventricular Dysfunction, Heart Failure and Cardiovascular Disease: CARDIA Study. The American Journal of Medicine. 2020; 133: 946-953.
[17] Gundersen T, Grøttum P, Pedersen T, Kjekshus JK. Effect of timolol on mortality and reinfarction after acute myocardial infarction: prognostic importance of heart rate at rest. The American Journal of Cardiology. 1986; 58: 20-24.

[18] Møller JE, Whalley GA, Dini FL, Doughty RN, Gamble GD, Klein AL, et al. Independent prognostic importance of a restrictive left ventricular filling pattern after myocardial infarction: an individual patient meta-analysis: Meta-Analysis Research Group in Echocardiography acute myocardial infarction. Circulation. 2008; 117: 2591-2598.

[19] Fox KM, Ferrari R. Heart rate: a forgotten link in coronary artery disease? Nature Reviews. Cardiology. 2011; 8: 369-379.

[20] Ohyama Y, Ambale-Venkatesh B, Noda C, Chugh AR, TeixidoTura G, Kim J, et al. Association of Aortic Stiffness with Left Ventricular Remodeling and Reduced Left Ventricular Function Measured by Magnetic Resonance Imaging: the Multi-Ethnic Study of Atherosclerosis. Circulation. Cardiovascular Imaging. 2016; 9: 10.1161/CIRCIMAGING.115.004426 e004426.

[21] Rossello X, Raposeiras-Roubin S, Latini R, DominguezRodriguez A, Barrabes JA, Sanchez PL, et al. Rationale and design of the pragmatic clinical trial tREatment with Beta-blockers after myOcardial infarction withOut reduced ejection fracTion (REBOOT). European Heart Journal - Cardiovascular Pharmacotherapy. 2021. (in press)

[22] Krumholz HM. Post-Hospital Syndrome - an Acquired, Transient Condition of Generalized Risk. New England Journal of Medicine. 2013; 368: 100-102.

[23] Mesquita ET, Cruz LN, Mariano BM, Jorge AJL. Post-Hospital Syndrome: a New Challenge in Cardiovascular Practice. Arquivos Brasileiros De Cardiologia. 2015; 105: 540-544.

[24] Tran H, Byatt N, Erskine N, Lessard D, Devereaux RS, Saczynski J, et al. Impact of anxiety on the post-discharge outcomes of patients discharged from the hospital after an acute coronary syndrome. International Journal of Cardiology. 2019; 278: 2833.

[25] Vazir A, Claggett B, Jhund P, Castagno D, Skali H, Yusuf S, et al. Prognostic importance of temporal changes in resting heart rate in heart failure patients: an analysis of the CHARM program. European Heart Journal. 2015; 36: 669-675.

[26] Matsukawa R, Masuda S, Matsuura H, Nakashima H, Ikuta H, Okabe $\mathrm{K}$, et al. Early follow-up at outpatient care after discharge improves long-term heart failure readmission rate and prognosis. ESC Heart Failure. 2021; 8: 3002-3013.

[27] Choe Y, Han J, Choi I, Park H. Changes in Oxygen Consumption and Heart Rate after Acute Myocardial Infarction during 6-Month Follow-up. PM \& R. 2018; 10: 587-593.

[28] Rodrigues B, Lira FS, Consolim-Colombo FM, Rocha JA, Caperuto EC, De Angelis K, et al. Role of exercise training on autonomic changes and inflammatory profile induced by myocardial infarction. Mediators of Inflammation. 2014; 2014: 702473.

[29] Lai Y, Huang C, Chang H, Chiu W, Tsai N, Cheng B, et al. Severity of Cardiovascular Autonomic Neuropathy is a Predictor Associated with Major Adverse Cardiovascular Events in Adults with Type 2 Diabetes Mellitus: a 6-Year Follow-up Study. Canadian Journal of Diabetes. 2021; 45: 155-161.

[30] Vinik AI, Casellini C, Parson HK, Colberg SR, Nevoret ML. Cardiac Autonomic Neuropathy in Diabetes: A Predictor of Cardiometabolic Events. Frontiers in Neuroscience. 2018; 12: 591.

[31] Lahiri MK, Kannankeril PJ, Goldberger JJ. Assessment of autonomic function in cardiovascular disease: physiological basis and prognostic implications. Journal of the American College of Cardiology. 2008; 51: 1725-1733.

[32] Wu X, Du R, Hu C, Cheng D, Ma L, Li M, et al. Resting heart rate is associated with metabolic syndrome and predicted 10-year risk of cardiovascular disease: a cross-sectional study. Journal of Diabetes. 2019; 11: 884-894. 